\title{
Transporte masa: Simulación de fluidos incomprensibles en una capa difusora de gas utilizando OpenFoam
}

\section{Mass transport: Simulation of incomprehensible fluids in a gas diffuser layer using OpenFoam}

PAMPLONA-SOLIS, Blandy†ं, CRUZ-ARGÜELLO, Julio*, GOMEZ-BARBA, Leopoldo y MAYCANCHE, Isaias

Universidad de Guadalajara - Centro Universitario de Ciencias Económico Administrativa

ID 1 ${ }^{\mathrm{er}}$ Autor: Blandy, Pamplona-Solis / ORC ID: 0000-0003-0293-992X, CVU CONACYT ID: 356408

ID $1^{\text {er }}$ Coautor: Julio, Cruz-Argüello / ORC ID: 0000-0001-8664-9422, CVU CONACYT ID: 221002

ID $2^{\text {do }}$ Coautor: Leopoldo, Gomez-Barba / ORC ID: 0000-0003-0608-3452, CVU CONACYT ID: 336905

ID $3^{\text {er }}$ Coautor: Isaias, May-Canche / ORC ID: 0000-0003-2897-6649, CVU CONACYT ID: 255571

\section{Resumen}

La Capa difusora de gases (GDL) provee una ruta de transporte para las especies reactantes desde los canales de flujo hasta la capa catalizadora en una celda de combustible. El diseño de los componentes requiere que las especies presenten una difusión uniforme hacia los electrodos y que los productos formados sean desalojados lo más rápido posible, para evitar el incremento de la resistencia óhmica debido al bloqueo de los sitios activos. Este trabajo presenta una propuesta de solución para la ecuación de continuidad en fluidos incomprensibles en un modelo 3D para una capa difusora de gases con características de malla de alambre para una celda de combustible utilizando el software de código abierto "OpenFoam". El objetivo del estudio es mostrar que bajo diferentes escalas de la capa difusora de gases se presentan cambios significativos en las velocidades de propagación de las especies reactantes hacia el área activa de la celda de combustible.

\begin{abstract}
The Gas Diffusion Layer (GDL) provides a transport route for the reactant species from the flow channels to the catalytic layer in a fuel cell. The components design requires that the species present a uniform diffusion towards the electrodes and that the formed products are shifted as faster as possible, to avoid the increase of the ohmic resistance due to the blocking of the active sites, or it constitutes a bottleneck that the performance decrease fast. This work presents a solution proposal for the continuity equation in incomprenssible fluids in a 3D model of a gas diffuser layer with titanium wired mesh characteristics of a fuel cell using "OpenFOAM" open source software. The aim of the study is to show that under different scales or size of the gas diffusion layer there are significant changes in the velocities of propagation of the reactant species towards the active area of the fuel cell.
\end{abstract}

Fuel Cell, GDL, OpenFoam

Citación: PAMPLONA-SOLIS, Blandy, CRUZ-ARGÜELLO, Julio, GOMEZ-BARBA, Leopoldo y MAY-CANCHE, Isaias. Transporte masa: Simulación de fluidos incomprensibles en una capa difusora de gas utilizando OpenFoam. Revista de Sistemas Experimentales. 2019. 6-20: 16-24

\footnotetext{
* Correspondencia al Autor (Correo electrónico: jcruz@itchetumal.edu.mx)

$\dagger$ Investigador contribuyendo como primer Autor
} 


\section{Introducción}

El estudio de la conversión directa de energía química a energía eléctrica por medio de celdas de combustible (Fuel Cell- FC, por sus siglas en inglés) ha ganado popularidad debido principalmente a dos factores: a) la alta eficiencia en la conversión de energía y b) la disminución de contaminantes emitidos a la atmósfera (Alvarado-Flores \& ÁvalosRodríguez, 2013; J. Wang, Yuan, Yu, \& Sunden, 2017; Wu, 2016).

El hidrógeno es el combustible principal de las celdas, sin embargo aunque es el elemento más abundante en el universo, no se encuentra en su estado puro en la naturaleza (Lodders, 2003), por lo que se requiere de procesos de reformado de hidrocarburos o electrolisis para su obtención. Durante su extracción a partir de combustibles fósiles se producen gases contaminantes que limita a las celdas de combustible como una fuente limpia de energía.

La electrolisis es un método limpio para obtener hidrógeno a través de la división de la molécula de agua. En este sentido se han creado Celdas de Combustible Regenerativas Unificadas (URFC - Unified Regenerative Fuel Cell), donde una misma unidad puede trabajar en modo celda de combustible para generar electricidad $\mathrm{o}$ en modo electrolizador para obtener hidrógeno. (Min, Ishida, Ito, \& Maeda, 2010; Y. Wang, Leung, Xuan, \& Wang, 2016).

La ventaja principal de las celdas de combustible es que no presentan la descarga espontánea de las baterías de ion de litio, ya que en cuanto se les suministra combustible (hidrógeno) generan energía, sin embargo es imperante, la búsqueda de materiales y diseños más eficientes para que la generación de energía de este tipo celdas pueda llegar a niveles de comercialización (Niu, Fan, Bao, \& Jiao, 2018). El negro carbón debido a su bajo costo, es el material ampliamente utilizado en los dos electrodos (ánodo y cátodo) en las celdas de combustible, por su parte la evolución del hidrógeno no presenta problemas de corrosión en la mayoría de los materiales (Gabbasa, Sopian, Fudholi, \& Asim, 2014), pero el proceso de evolución del oxígeno en el electrolizador origina serios problemas de corrosión a causa de los sobrepotenciales anódicos (Kim, Lee, \& Tak, 2009; Min et al., 2010).
El desarrollo de un electrodo para el modo electrolizador es intrínsecamente diferente al de la celda de combustible, pues en ambos casos es necesario un adecuado equilibrio de las propiedades hidrofóbicas e hidrófilas. (Ma, Sui, \& Zhai, 2008; Pettersson, Ramsey, \& Harrison, 2006).

Un adecuado diseño de la celda de combustible requiere que las especies presenten una difusión uniforme hacia los electrodos y que los productos resultantes del proceso electroquímico sean desalojados lo más rápido posible para evitar inundación en la celda; sin embargo, para su optimización es necesario saber cómo ocurre la distribución de las especies reactivas en los canales de flujo, así como la difusión de las mismas sobre las capas de soporte mediante el estudio de la mecánica de fluidos, por lo que la simulación de modelos que puedan predecir su funcionamiento es esencial y evitar los problemas de las técnicas experimentales que se presentan en laboratorio (Aguirre, 2009; Yin, $\mathrm{Wu}, \mathrm{He}, \mathrm{Du}, \&$ Jiao, 2014).

La Capa difusora de gases (GDL - Gas Diffusion Layer) provee una ruta para los gases reactantes desde los canales de flujo hasta la capa catalizadora, permitiendo el acceso al área activa, por lo que es importante conocer su comportamiento dentro del funcionamiento de las FC. (Ionescu \& Buzbuchi, 2017; J. Wang et al., 2017; Yin et al., 2014)

Las técnicas experimentales para mejorar las condiciones de generación de electricidad, así como la producción de hidrógeno son usualmente costosas y prolongadas. Los modelos matemáticos con la simulación han sido utilizados como herramientas en el proceso de descubrimiento de nuevos conocimientos en el diseño de las celdas de combustible. En la literatura existen múltiples trabajos para modelar las FC, sin embargo, existen pocos estudios enfocados a las Celdas Regenerativas Unificadas y mucho menos a modelos del comportamiento de la Capa Difusora de Gases (Abdol Rahim, Tijani, Kamarudin, \& Hanapi, 2016; Colleen Spiegel, 2008).

Los resultados reportados en modelos de simulación de celdas de combustible consideran a la GDL como un medio poroso, descartando su composición geométrica a nivel macroscópico (Owejan, Trabold, \& Mench, 2014; Truc, Ito, \& Fushinobu, 2018; Wan et al., 2018; J. Wang et al., 2017; Zhang, Cheng, \& He, 2017).

PAMPLONA-SOLIS, Blandy, CRUZ-ARGÜELLO, Julio, GOMEZBARBA, Leopoldo y MAY-CANCHE, Isaias. Transporte masa: Simulación de fluidos incomprensibles en una capa difusora de gas utilizando OpenFoam. Revista de Sistemas Experimentales. 2019 
Por lo que en este trabajo se considera importante conocer el efecto de la capa difusora con diferentes geometrías en el transporte de los gases en su ruta hacia el catalizador durante el modo electrolizador en el cátodo de la celda.

La GDL en una URFC tiene un funcionamiento diferente al que tiene en una Celda de Combustible. A través de la Capa Difusora los gases humidificados mantienen húmeda la membrana tanto de la FC como de la operación de la URFC en modo celda de combustible; sin embargo, el exceso de agua en las rutas de trasporte de gas de la GDL, especialmente el electrodo de oxígeno (cátodo durante modo celda de combustible), induce a la pérdida de transporte de masa (Gabbasa et al., 2014).

Aunque las celdas de combustible están integradas con componentes fijos, es decir, no tienen partes movibles o motores para funcionar (Fig. 1). El fenómeno que sucede en su interior es complejo, debido a que más de un proceso sucede en el mismo momento, es el caso de la GDL que debe ser optimizada para que el gas reactante circule eficientemente, al mismo tiempo que el agua debe viajar en dirección opuesta y no acumularse en los espacios de circulación, además que debe ser conductiva eléctrica y térmicamente (Al-baghdadi, 2017).

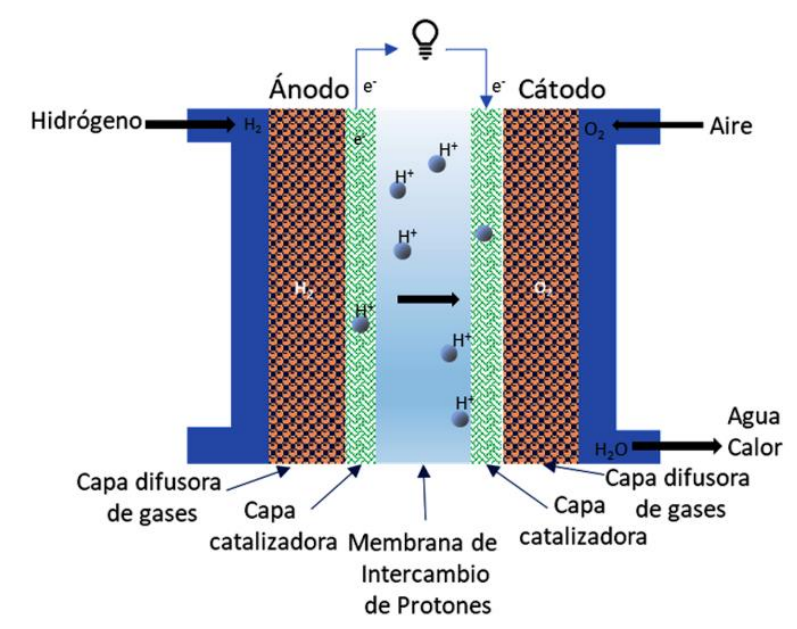

Figura 1 Componentes de una celda de combustible

\section{Método}

Este trabajo desarrolla tres casos de simulaciones en un ambiente tridimensional (3D) del transporte del oxígeno a través de mallas de titanio tipo tela utilizadas como capa difusora de gases. Las características de la tela de titanio comercializada por la empresa Fuel Cell Store son mostradas en la Tabla 1(Store, n.d.).

\begin{tabular}{|c|c|c|c|}
\hline $\begin{array}{c}\text { Código } \\
\text { Producto }\end{array}$ & Espesor & $\begin{array}{c}\text { Grosor } \\
\text { Hilo }\end{array}$ & $\begin{array}{c}\text { Longitud } \\
\text { Diamante }\end{array}$ \\
\hline $592779 \quad \&$ & & & \\
592780 & $50.8 \mu \mathrm{m}$ & $101.6 \mu \mathrm{m}$ & $787 \mu \mathrm{m}$ \\
\hline
\end{tabular}

Tabla 1 Especificación de la malla

Durante el proceso de simulación un paso muy importante es la creación de la geometría del sistema a modelar por medio de software CAD (computer-aided design, por sus siglas en inglés), la especificación de las dimensiones de cada elemento con forma de diamante de la mallada de titanio tipo tela es presentada en la Fig. 2.

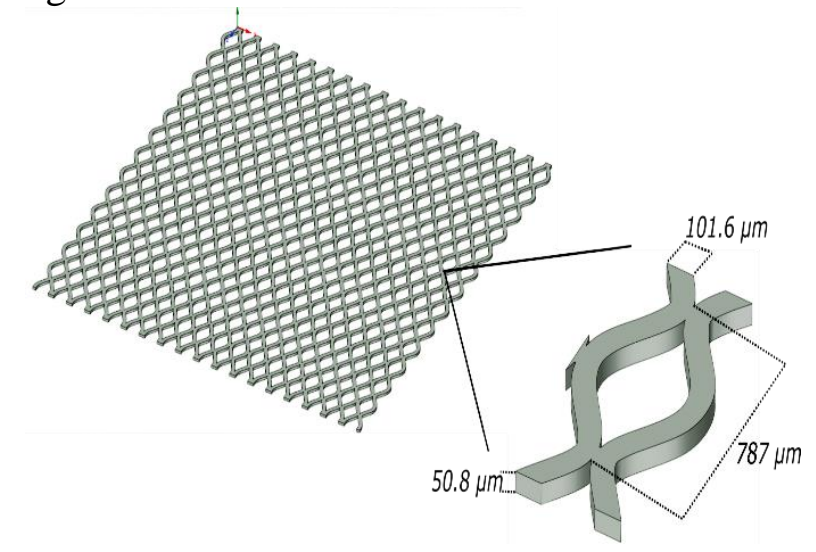

Figura 2 Especificación del diamante de la malla utilizada en la GDL

Para la simulación de los tres casos se modificó la geometría de la GDL por medio de la colocación de tres mallas de titanio tipo tela en diferentes posiciones: a) caso 1 mallas colocadas en $0^{\circ}-0^{\circ}-0^{\circ}$, b) caso 2 mallas colocadas en $0^{\circ}-90^{\circ}-0^{\circ}$ y c) caso 3 mallas $0^{\circ}$ $45^{\circ}-0^{\circ}$. Las cuáles deben ser replicables al momento de ensamblar la celda de combustible en laboratorio con el objetivo de validar los resultados de la simulación. La Fig. 3 presenta las tres configuraciones planteadas en esta investigación.

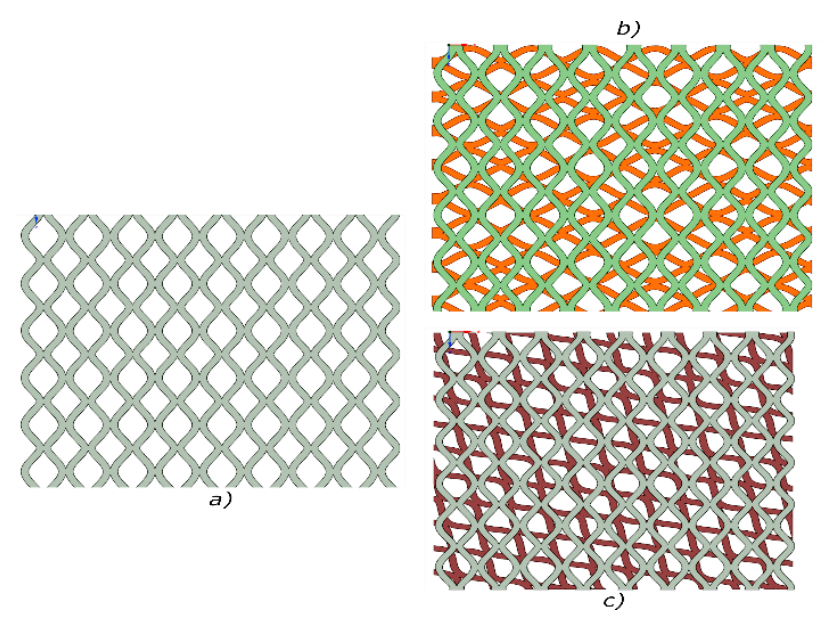

Figura 3 Colocación de las mallas: a) $0^{\circ}-0^{\circ}-0^{\circ}$ b) $0^{\circ}-$ $90^{\circ}-0^{\circ}$ c) $0^{\circ}-45^{\circ}-0^{\circ}$

PAMPLONA-SOLIS, Blandy, CRUZ-ARGÜELLO, Julio, GOMEZBARBA, Leopoldo y MAY-CANCHE, Isaias. Transporte masa: Simulación de fluidos incomprensibles en una capa difusora de gas utilizando OpenFoam. Revista de Sistemas Experimentales. 2019 


\section{Ecuaciones que gobiernan el fenómeno}

El comportamiento de las especies que son transportadas dentro de la celda de combustible es modelado por medio de las ecuaciones de conservación. Ecuación de continuidad en forma diferencial:

$\frac{\partial \rho}{\partial t}+\frac{\partial(\rho u)}{\partial x}+\frac{\partial(\rho v)}{\partial y}+\frac{\partial(\rho w)}{\partial z}=0$

\section{Donde:}

$t$ es el tiempo

$\rho$ es la densidad

$u, v, w$ son las velocidades en $X, Y, Z$

La ecuación puede ser expresada en su forma vectorial:

$$
\frac{\partial \rho}{\partial t}+? .(\rho \vec{V})=0
$$

La ecuación de momento puede ser escrita para cada una de las direcciones, las cuales son llamadas ecuaciones de Navier Stokes.

$\frac{\partial \vec{V}}{\partial t}+(\vec{V} . ?) \vec{V}=-\frac{1}{\rho}$ ? $p+v\left(?^{2} \vec{V}\right)$

Donde:

$p$ es la presión

$\vec{V}$ es el vector de velocidades

\section{Descripción del modelo}

El espacio geométrico utilizado en la simulación para el transporte del fluido a través del cátodo es presentado en la Fig. 4, está compuesto por tres elementos principales: 1) el serpentín (elemento color gris), 2) la capa difusora de gases (compuesta por las tres telas de malla de titanio, elemento en color verde) y 3) el catalizador (elemento color azul).

El modelo tridimensional del sistema establece una velocidad de entrada (INLET = $0.05 \mathrm{~m} / \mathrm{s}$ en dirección al eje y), con paredes no deslizables y una presión relativa de salida (OUTLET $=0=110325$ pascales). Los valores utilizados como condiciones de frontera y geometría se especifican en la tabla 2.

\section{Supuestos del modelo}

Para la ejecución de las simulaciones del modelo $3 \mathrm{D}$, se configura como un sistema de fase simple, isotérmico y de estado estable, asumiendo:

1. La temperatura dentro de la celda es uniforme con un valor constante de $298.15^{\circ} \mathrm{K}$. No se considera algún intercambio de calor.

2. El número de Reynolds y la velocidad son bajas, por lo que prevalecen condiciones de flujo laminar.

3. La PEM es impermeable a los gases.

4. Los gases son considerados incomprensibles.

5. La presión en las fronteras se establece con el valor de presión atmosférica.

6. La GDL es una malla regular.

\section{Experimentación}

La primera etapa del procedimiento es el preprocesamiento, en la cual se fijan el dominio, los principios y supuestos del problema físico. La siguiente fase que se realiza es la definición del modelo matemático que gobernará el comportamiento entre las variables durante el proceso de simulación, así mismo se debe elegir el método de solución.

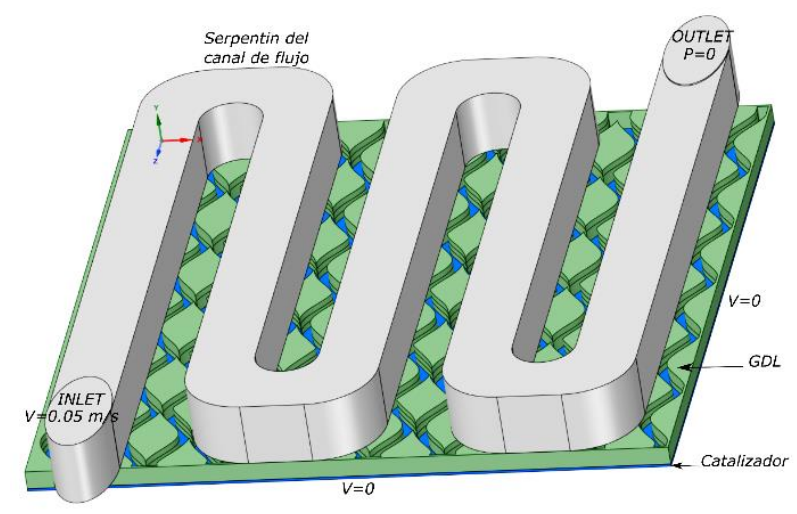

Figura 4 Contexto del modelo

\begin{tabular}{|l|r|l|}
\hline Parámetro & Valor & Unidades \\
\hline Altura canal serpentin & 0.5 & $\mathrm{~mm}$ \\
\hline Ancho canal serpentin & 0.5 & $\mathrm{~mm}$ \\
\hline Espaciamiento entre canales & 0.5 & $\mathrm{~mm}$ \\
\hline Espesor de la capa difusora & 0.15 & $\mathrm{~mm}$ \\
\hline Espesor de la capa catalítica & 0.03 & $\mathrm{~mm}$ \\
\hline Área de la capa difusora & 25 & $\mathrm{~mm}^{2}$ \\
\hline Velocidad de entrada & 0.05 & $\mathrm{~m} / \mathrm{s}$ \\
\hline Presión salida & 110325 & $\mathrm{pascal}$ \\
\hline
\end{tabular}

Tabla 2 Condiciones del contexto del modelo

PAMPLONA-SOLIS, Blandy, CRUZ-ARGÜELLO, Julio, GOMEZBARBA, Leopoldo y MAY-CANCHE, Isaias. Transporte masa: Simulación de fluidos incomprensibles en una capa difusora de gas utilizando OpenFoam. Revista de Sistemas Experimentales. 2019 
La solución de las ecuaciones que gobiernan la mecánica de fluidos se realizó por medio del método numérico de volúmenes finitos implementados en el CFD (Computational Fluid Dynamics) de código abierto denominado OpenFoam (Martin, 2019). Para la utilización de este método se realizó el diseño de la geometría definida como en la Fig. 4 , en este proceso se utilizó software CAD para facilitar su creación.

Una vez realizada la geometría, se subdividió el dominio en volúmenes de control por medio de mallados obteniendo el grid computacional que se esboza en la Fig. 5. Debido a la diferencia en los tamaños de los elementos que componen el modelo (serpentin, GDL y catalizador) se aplicó un mallado no uniforme con celdas principalmente tetraédricas que son más adecuadas en geometrías complejas, pero con la desventaja de mayores tiempos de ejecución para encontrar la convergencia en la solución de las ecuaciones.

Es importante señalar que en el proceso de mallado se debe mantener la continuidad del grid computacional entre los diferentes elementos que integran al modelo completo (serpentín, GDL y catalizador). Así mismo durante el proceso de mallado se requiere definir las zonas de frontera para establecer los parámetros de ejecución de la simulación.

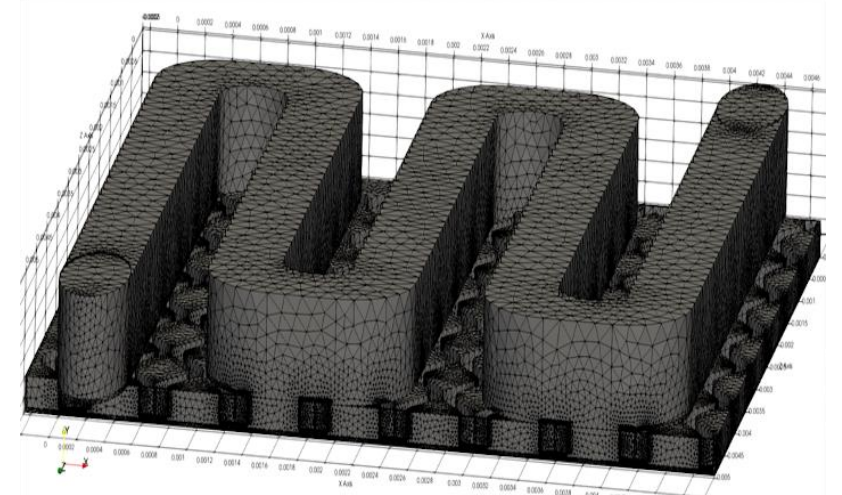

Figura 5 Grid de celdas obtenido del proceso de mallado

El grid con mayor número de celdas fue el del caso $2\left(0^{\circ}-45^{\circ}-0^{\circ}\right)$ con un total de $1,684,409$ elementos usados para simular el transporte del oxígeno en el cátodo de celda de combustible. El algoritmo de solución usado fue el algoritmo SIMPLE (OpenFOAM, n.d.), configurado para tener una convergencia en 10 e-6 en las variables de continuidad y velocidad.

\section{Resultados y discusión}

Por medio de la ejecución del algoritmo SIMPLE se encontró la convergencia en los tres casos de GDL presentados en este trabajo. Los resultados de la simulación en openFoam son guardadas en los directorios de tiempo que son utilizados posteriormente para la fase de postprocesamiento.

\section{Caso 1 GDL $0^{\circ}-0^{\circ}-0^{\circ}$}

La simulación se realizó con tres mallas todas colocadas en posición de cero grados, como se presenta en la Fig. 3a. Los resultados obtenidos son procesados con el software open-source de visualización Paraview, la mayor velocidad es alcanzada en el centro del serpentín con $1.5 \mathrm{e}-1$ m/s, como se observa en la Fig. 6.

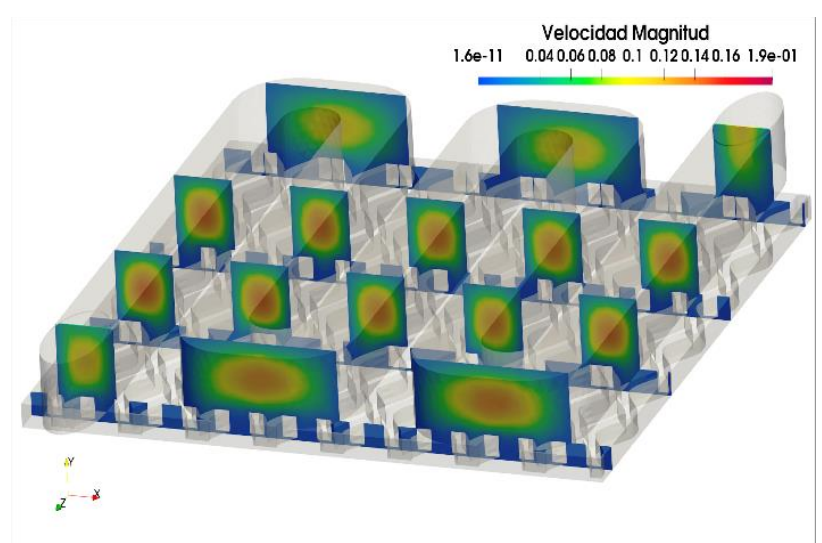

Figura 6 Perfiles de velocidad caso 1

Respecto al transporte del oxígeno en la zona de la capa difusora de gases, se presenta una distribución del fluido únicamente sobre el área de contacto con el serpentín, sin tener un movimiento considerable hacia otros sitios rumbo al catalizador (Fig. 7), las velocidades alcanzadas en este segmento de la celda se encontraron en un rango de $8.2104 \mathrm{e}-08$ hasta $0.043753 \mathrm{~m} / \mathrm{s}$.

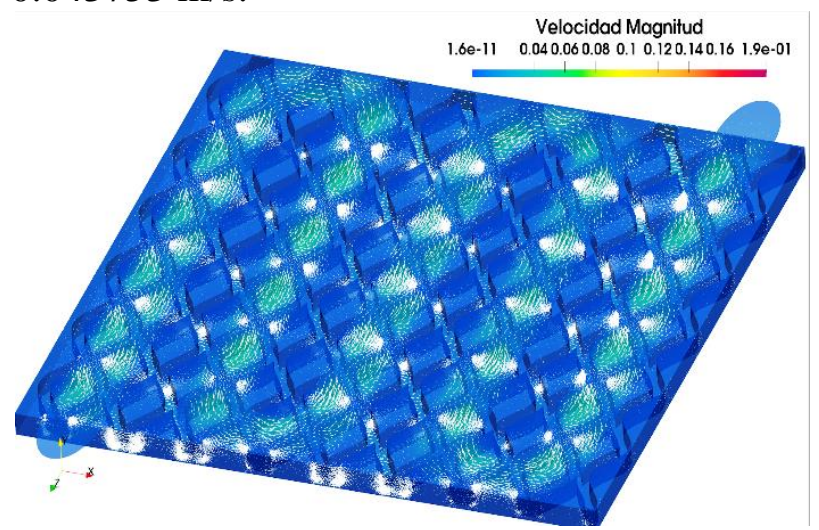

Figura 7 Vectores de velocidad GDL caso 1 


\section{Caso 2 GDL alternado $0^{\circ}-\mathbf{9 0}^{\circ}-0^{\circ}$}

Este caso de estudio consistió en modelar la capa difusora alternando la colocación de las tres mallas bajo el siguiente orden $0-90$ - 0 grados. El comportamiento presentado en el serpentín fue el esperado al concentrarse las mayores velocidades en el área central del flujo con velocidades de $2.1 \mathrm{e}-1 \mathrm{~m} / \mathrm{s}$ (Fig. 8 ).

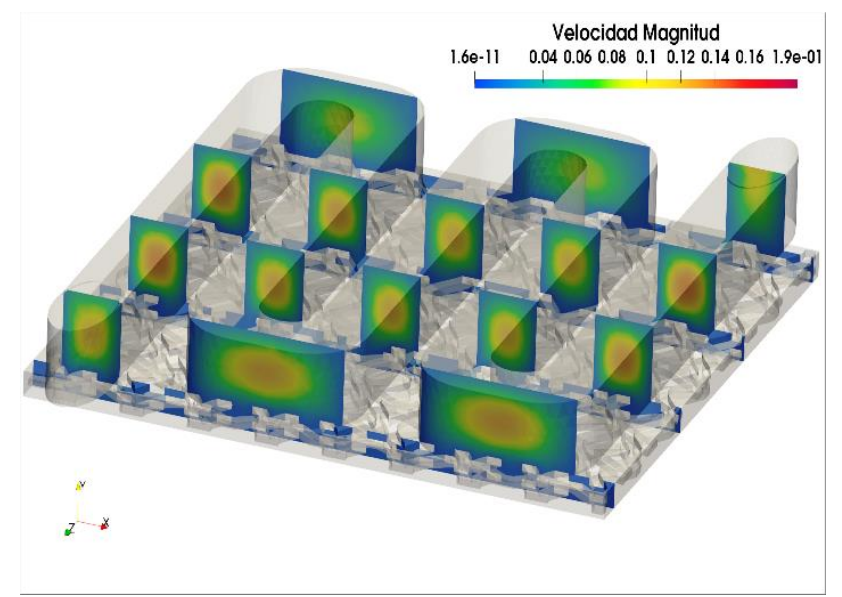

Figura 8 Perfiles de velocidad caso 2

La distribución del fluido en la GDL y catalizador exhibe un comportamiento más errático con las zonas de contacto, el choque de las moléculas de oxígeno con la disposición de los bordes de la GDL logran que haya mayor distribución en la capa del catalizador. En el área presentada en la Fig. 9 se obtuvieron velocidades entre $1.4165 \mathrm{e}-11$ y $8.227 \mathrm{e}-1 \mathrm{~m} / \mathrm{s}$.

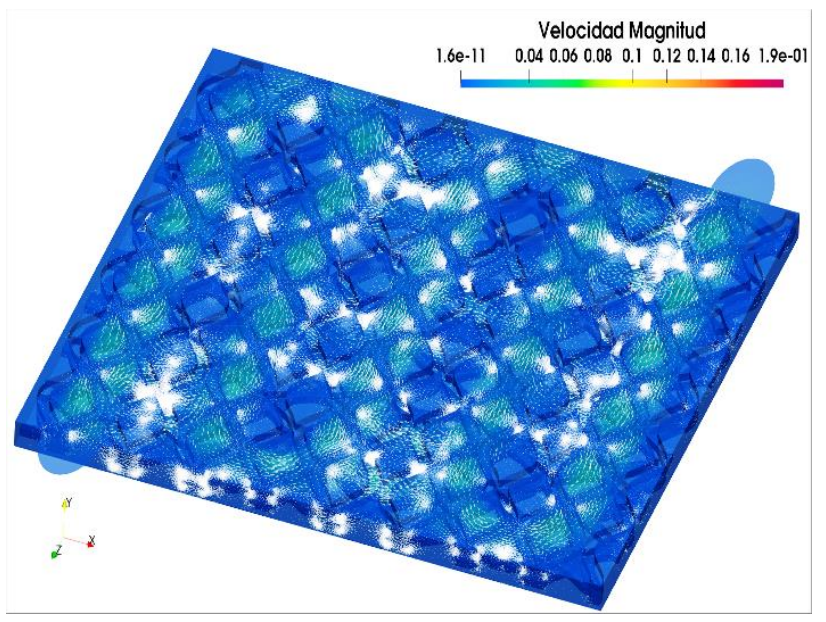

Figura 9 Vectores de velocidad GDL caso 2

Caso 3 GDL alternado $0^{\circ}-45^{\circ}-0^{\circ}$

La ultima configuración de la capa difusora se realizó ubicando alternadamente las mallas en las posiciones de $0-45-0$ grados.
La mayor velocidad alcanzada en el centro del serpentín fue de $1.5 \mathrm{e}-1 \mathrm{~m} / \mathrm{s}$, al igual que en los casos anteriores el comportamiento del flujo del oxígeno en esta zona fue similar (Fig. 10).

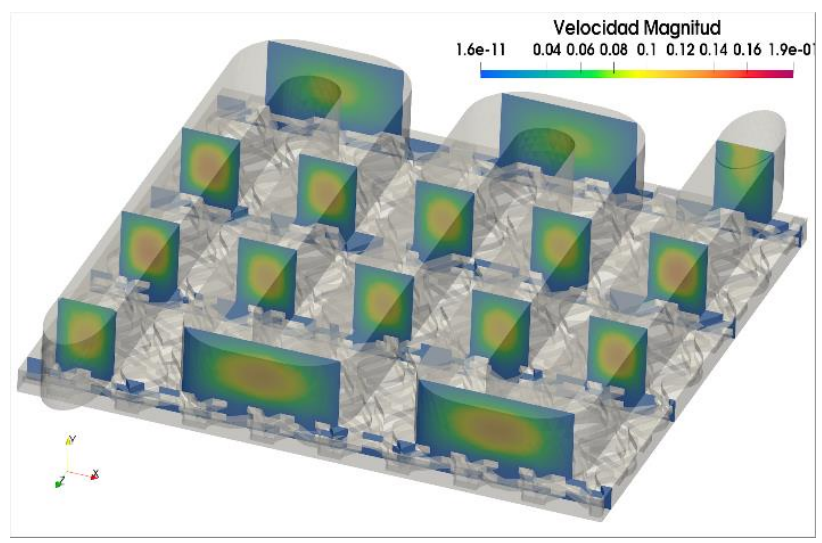

Figura 10 Perfiles de velocidad caso 3

El flujo del oxígeno en la zona del GDL para el caso 3 tuvo mayor distribución hacia el catalizador como se observa en la Fig. 10, sin embargo las velocidades obtenidas oscilan entre $3.234471 \mathrm{e}-10$ y $9.4474 \mathrm{e}-2 \mathrm{~m} / \mathrm{s}$ siendo las menores de los tres casos analizados (Fig. 11).

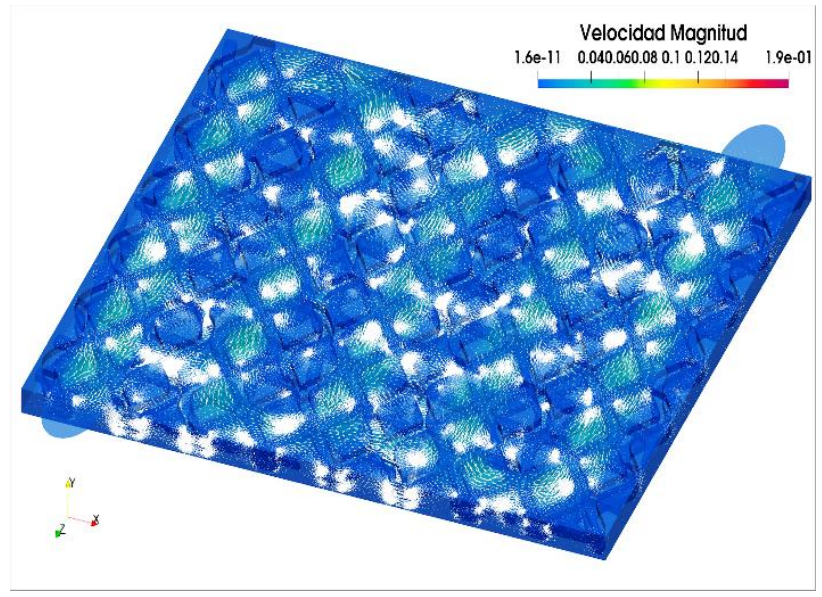

Figura 11 Vectores de velocidad GDL caso 3

En este trabajo la Capa Difusora de Gases es considerada como una malla regular de titanio lo que permite analizar el flujo de los reactantes a escala macroscópica la cual se fundamenta en el transporte por convección y como el cambio en la geometría favorece al flujo de las especies, a diferencia de la mayoría de las investigaciones que establecen a la GDL como un medio poroso para lo cual se establece un índice de difusión omitiendo la forma de los espacios creados en la GDL. 
Los CFD son herramientas computacionales que se utilizan ampliamente para simular la dinámica de fluidos en un vasto número de aplicaciones incluidas entre ellas la simulación en las celdas de combustible, en la actualidad se puede encontrar en el mercado software CFD propietario (no software libre) (ANSYS, COMSOL, Autodesk CFD, etc) con costos elevados de licenciamiento, por lo que utilizarlo en proyectos de investigación es necesario contar con el presupuesto suficiente para su adquisición, debido a esto es importante incursionar en el uso de software open-source que permita aplicar las ventajas de los CFD en la investigación sin que los recursos económicos sean una limitante.

La aplicación del software OpenFoam en la simulación del comportamiento de los fluidos en las celdas de combustible apoyará a nuevos investigadores demostrando la potencialidad y confiabilidad de las herramientas open-source.

\section{Conclusiones}

El objetivo de este trabajo es analizar los cambios que presenta la velocidad durante el fenómeno de transporte del oxígeno en su ruta desde la placa difusora de flujo (serpentín) hacia el catalizador en una celda de combustible, con base a la realización de tres simulaciones con configuraciones diferentes en la colocación de la tela mallada de titanio a diferentes grados de solapamiento.

En cada uno de los casos se realizó la configuración de las condiciones de frontera y configuraciones en el solver simpleFoam establecido para flujos incompresibles descartando las opciones de turbulencia que permite trabajar en OpenFoam.

Después de la ejecución de las simulaciones se obtuvieron las velocidades del flujo del oxígeno que se presentan en la tabla 3 , encontrándose que el caso 2 presenta la mayor velocidad de transporte con $8.227 \mathrm{e}-1 \mathrm{~m} / \mathrm{s}$, mientras que la configuración $(0-0-0)$ tiene la menor con $4.3753 \mathrm{e}-2 \mathrm{~m} / \mathrm{s}$. Asimismo la mayor velocidad obtenida en el cátodo fue obtenida en el caso 2 con $2.1 \mathrm{e}-1 \mathrm{~m} / \mathrm{s}$ a diferencia de $1.5 \mathrm{e}-1$ $\mathrm{m} / \mathrm{s}$ hallada en los casos 1 y 3 .
A partir de los resultados generados se puede establecer que la disposición geométrica de la capa difusora de gases (GDL) contribuye a cambios en la distribución y las velocidades del flujo de los gases dentro de la celda de combustible, lo cual es una condicionante del rendimiento de la celda.

\begin{tabular}{|c|c|c|c|}
\hline Caso estudio & $\begin{array}{l}\text { Velocidad } \\
\text { cátodo } \\
(\mathrm{m} / \mathrm{s})\end{array}$ & $\begin{array}{c}\text { Velocidad } \\
\text { GDL } \\
(\mathrm{m} / \mathrm{s})\end{array}$ & $\begin{array}{c}\text { Número de } \\
\text { celdas grid } \\
\text { computacional }\end{array}$ \\
\hline $\begin{array}{l}\text { Caso 1, } \\
\text { solapamiento } \\
(0-0-0)\end{array}$ & $1.5 \mathrm{e}-1$ & $\begin{array}{l}8.2104 \mathrm{e}- \\
08 \quad a \\
4.3753 \mathrm{e}-2\end{array}$ & 1159792 \\
\hline $\begin{array}{l}\text { Caso 2, } \\
\text { solapamiento } \\
(0-90-0)\end{array}$ & $2.1 \mathrm{e}-1$ & $\begin{array}{l}1.4165 \mathrm{e}- \\
11 \\
8.227 \mathrm{e}-1\end{array}$ & 1602683 \\
\hline $\begin{array}{l}\text { Caso } 3, \\
\text { solapamiento } \\
(0-45-0)\end{array}$ & $1.5 \mathrm{e}-1$ & $\begin{array}{l}3.23447 \mathrm{e}- \\
10 \quad a \quad a \\
9.4474 \mathrm{e}-2 \\
\end{array}$ & 1684409 \\
\hline
\end{tabular}

Tabla 3 Resultado de la velocidad en casos de estudio

El proceso de transporte en una URFC involucra la entrada y salida del hidrógeno, oxígeno y agua dependiendo del modo de funcionamiento de la celda, por lo que es importante ampliar el alcance de la investigación para conocer el efecto de la geometría en las velocidades al introducir líquidos y durante el cambio de fase líquida a gaseosa de los compuestos químicos.

\section{Agradecimientos}

Agradecemos el apoyo brindado por el Doctorado en Tecnologías de Información de la Universidad de Guadalajara y al Instituto Tecnológico de Chetumal. Este trabajo es soportado por el proyecto de ciencia básica 235848: "Estudio y Desarrollo de la Capa Difusora de Gas/Líquido de una Celda de Combustible Regenerativa Unificada tipo PEM". CONACYT 2015-2018.

\section{Referencias}

Abdol Rahim, A. H., Tijani, A. S., Kamarudin, S. K., \& Hanapi, S. (2016). An overview of polymer electrolyte membrane electrolyzer for hydrogen production: Modeling and mass transport. Journal of Power Sources, 309, 5665 .

https://doi.org/10.1016/j.jpowsour.2016.01.012 
Aguirre, A. (2009). Descripción y Modelado de una Pila de Combustible de Membrana de Intercambio Protónico, 231. Retrieved from http://e-

archivo.uc3m.es/bitstream/handle/10016/6055/ PFC_Antonio_Mayandia_V2.pdf?sequence $=1$

Al-baghdadi, M. A. R. S. (2017). Proton exchange membrane fuel cells modeling: A review of the last ten years results of the Fuel Cell Research Center-IEEF. International Journal of Energy and Environment, 8(1), 1-28.

Alvarado-Flores, J., \& Ávalos-Rodríguez, L. (2013). Materiales para ánodos, cátodos y electrolitos utilizados en celdas de combustible de óxido sólido. Revista Mexicana de Fisica, 59(1), 66-87.

Colleen Spiegel. (2008). PEM Fuel Cell: Modeling and Simulation using MATLAB. (Elsiever, Ed.). Elsiever. https://doi.org/10.1016/B978-0123742599.50006-9

Gabbasa, M., Sopian, K., Fudholi, A., \& Asim, N. (2014). A review of unitized regenerative fuel cell stack: Material, design and research achievements. International Journal of Hydrogen Energy, 39(31), 17765-17778. https://doi.org/10.1016/j.ijhydene.2014.08.121

Ionescu, V., \& Buzbuchi, N. (2017). PEMFC Two-dimensional FEM Model to Study the Effects of Gas Flow Channels Geometry on Reactant Species Transport. Energy Procedia, 112(October 2016), 390-397. https://doi.org/10.1016/j.egypro.2017.03.1085

Kim, J., Lee, J., \& Tak, Y. (2009). Relationship between carbon corrosion and positive electrode potential in a proton-exchange membrane fuel cell during start/stop operation. Journal of Power Sources, 192(2), 674-678. https://doi.org/10.1016/J.JPOWSOUR.2009.03. 039

Lodders, K. (2003). SOLAR SYSTEM ABUNDANCES AND CONDENSATION TEMPERATURES OF THE ELEMENTS Katharina Lodders. Sciences-New York, 591(2), 1220-1247. Retrieved from http://iopscience.iop.org/0004-

637X/591/2/1220
Ma, L., Sui, S., \& Zhai, Y. (2008). Preparation and characterization of $\mathrm{Ir} / \mathrm{TiC}$ catalyst for oxygen evolution. Journal of Power Sources, $177(2)$, 470-477. https://doi.org/10.1016/j.jpowsour.2007.11.106

Martin, J. (2019). Universidad nacional del santa.

Min, C., Ishida, M., Ito, H., \& Maeda, T. (2010). Influence of properties of gas diffusion layers on the performance of polymer electrolyte-based unitized reversible fuel cells. International Journal of Hydrogen Energy, 36(2), 1740-1753. https://doi.org/10.1016/j.ijhydene.2010.10.091

Niu, Z., Fan, L., Bao, Z., \& Jiao, K. (2018). Numerical investigation of innovative 3D cathode flow channel in proton exchange membrane fuel cell. International Journal of Energy Research, 42(10), 3328-3338. https://doi.org/10.1002/er.4086

OpenFOAM. (n.d.). OpenFOAM: User Guide: SIMPLE algorithm. Retrieved March 11, 2019, from https://www.openfoam.com/documentation/gui des/latest/doc/guide-applications-solverssimple.html

Owejan, J. P., Trabold, T. A., \& Mench, M. M. (2014). Oxygen transport resistance correlated to liquid water saturation in the gas diffusion layer of PEM fuel cells. International Journal of Heat and Mass Transfer, 71, 585-592. Retrieved from http://dx.doi.org/10.1016/j.ijheatmasstransfer.2 013.12.059

Pettersson, J., Ramsey, B., \& Harrison, D. (2006). A review of the latest developments in electrodes for unitised regenerative polymer electrolyte fuel cells. Journal of Power Sources, 157(1), 28-34. https://doi.org/10.1016/j.jpowsour.2006.01.059

Store, F. C. (n.d.). Titanium Screen. Retrieved February 8, 2019, from https://www.fuelcellstore.com/fuel-cellcomponents/gas-diffusion-layers/wire-meshand-cloth/titanium-screen-mesh-cloth 
Truc, N. T., Ito, S., \& Fushinobu, K. (2018). Numerical and experimental investigation on the reactant gas crossover in a PEM fuel cell. International Journal of Heat and Mass Transfer, 127, 447-456. https://doi.org/10.1016/j.ijheatmasstransfer.201 8.07 .092

Wan, Z. H., Zhong, Q., Liu, S. F., Jin, A. P., Chen, Y. N., Tan, J. T., \& Pan, M. (2018). Determination of oxygen transport resistance in gas diffusion layer for polymer electrolyte fuel cells. International Journal of Energy Research, 42(6), 2225-2233.

https://doi.org/10.1002/er.4012

Wang, J., Yuan, J., Yu, J.-S., \& Sunden, B. (2017). Investigation of effects of nonhomogenous deformation of gas diffusion layer in a PEM fuel cell. International Journal of Energy Research, 41(14), 2121-2137. Retrieved from http://dx.doi.org/10.1002/er.3774

Wang, Y., Leung, D. Y. C., Xuan, J., \& Wang, H. (2016). A review on unitized regenerative fuel cell technologies, part-A: Unitized regenerative proton exchange membrane fuel cells. Renewable and Sustainable Energy Reviews, 65, 961-977. https://doi.org/10.1016/j.rser.2016.07.046

$\mathrm{Wu}, \mathrm{H}$. (2016). A review of recent development : Transport and performance modeling of PEM fuel cells. Applied Energy, 165, 81-106. https://doi.org/10.1016/j.apenergy.2015.12.075

Yin, Y., Wu, T., He, P., Du, Q., \& Jiao, K. (2014). Numerical simulation of two-phase cross flow in microstructure of gas diffusion layer with variable contact angle. International Journal of Hydrogen Energy, 39(28), 1577215785.

https://doi.org/10.1016/j.ijhydene.2014.07.162

Zhang, Z., Cheng, J., \& He, X. (2017). Numerical simulation of flow and heat transfer in composite PCM on the basis of two different models of open-cell metal foam skeletons. International Journal of Heat and Mass Transfer.

https://doi.org/10.1016/j.ijheatmasstransfer.201 7.05 .012 